\title{
Capacity gaps hinder the performance of marine protected areas globally
}

\author{
Authors: David A. Gill ${ }^{12^{*}}$, Michael B. Mascia ${ }^{3}$, Gabby N. Ahmadia ${ }^{4}$, Louise Glew ${ }^{4}$, Sarah E. \\ Lester $^{5}$, Megan Barnes ${ }^{6}$, Ian Craigie ${ }^{7}$, Emily Darling ${ }^{8}$, Christopher M. Free ${ }^{9}$, Jonas \\ Geldmann ${ }^{10,11}$, Susie Holst ${ }^{12}$, Olaf P. Jensen ${ }^{9}$, Alan T. White ${ }^{13}$, Xavier Basurto ${ }^{14}$, Lauren Coad ${ }^{15}$, \\ Ruth D. Gates ${ }^{16}$, Greg Guannel ${ }^{17}$, Peter J. Mumby ${ }^{18}$, Hannah Thomas ${ }^{19}$, Sarah Whitmee ${ }^{20}$, \\ Stephen Woodley ${ }^{21}$ and Helen E. Fox ${ }^{4,22}$
}

\begin{abstract}
Amidst a proliferation of marine protected areas (MPAs) to conserve marine resources, it is unclear whether many MPAs are being effectively and equitably managed, and how MPA management influences substantive outcomes. We developed a global database of management and fish population data (433 and 218 MPAs, respectively) to assess: 1) MPA management processes; 2) MPA impacts on fish populations, and; 3) relationships between management processes and ecological impacts. Many MPAs failed to meet thresholds for effective and equitable management processes, with widespread shortfalls in staff and financial resources. Although $71 \%$ of MPAs positively impacted fish populations, these conservation impacts were highly variable. Staff and budget capacity were the strongest predictors of conservation impacts: MPAs with adequate staff capacity had ecological impacts 2.9 times greater than MPAs with inadequate capacity. Thus, continued global expansion of MPAs without adequate investment in human and financial capacity will likely lead to sub-optimal conservation outcomes.
\end{abstract}

Keywords: Marine protected areas, Convention on Biological Diversity, impact evaluation, common pool resources, biodiversity conservation, fisheries.

\footnotetext{
Affiliations:

${ }^{1}$ National Socio-Environmental Synthesis Center (SESYNC), Annapolis, Maryland 21401. ${ }^{2}$ Luc Hoffmann Institute, World Wildlife Fund International, 1196 Gland, Switzerland. ${ }^{3}$ Moore Center for Science, Conservation International, Arlington, Virginia 22202 USA. ${ }^{4}$ World Wildlife Fund US, Washington DC 20037 USA. ${ }^{5}$ Department of Geography, Florida State University, Florida 32306 USA. ${ }^{6}$ Centre for Biodiversity and Conservation Science, University of Queensland, St Lucia Campus, Brisbane, Qld 4072, Australia. ${ }^{7}$ ARC Centre of Excellence for Coral Reef Studies, James Cook University, Townsville, Qld 4811, Australia. ${ }^{8}$ Wildlife Conservation Society, The Bronx, NY USA. ${ }^{9}$ Department of Marine \& Coastal Sciences, Rutgers University, New Brunswick, NJ 08901 USA. ${ }^{10}$ Conservation Science Group, Department of Zoology, University of Cambridge, Downing Street, Cambridge CB2 3EJ, UK. ${ }^{11}$ Center for Macroecology, Evolution and Climate, Natural History Museum of Denmark, University of Copenhagen, Universitetsparken 15, DK-2100 Copenhagen E, Denmark. ${ }^{12}$ NOAA Coral Reef Conservation Program, Silver Spring, Maryland 20910 USA. ${ }^{13}$ Indo-Pacific Division, The Nature Conservancy, Honolulu, Hawaii, USA. ${ }^{14}$ Nicholas School of the Environment, Duke University, Beaufort, NC, 28516, USA. ${ }^{15}$ CIFOR - Center for International Forestry Research, University of Oxford, Oxford, UK. ${ }^{16}$ Hawaii Institute of Marine Biology, University of Hawaii at Manoa, Hawaii 96744 USA. ${ }^{17}$ The Natural Capital Project, Stanford University, 371 Serra Mall, Stanford, California 94305-5020, USA. ${ }^{18}$ Marine Spatial Ecology Lab, School of Biological Sciences and ARC Centre of Excellence for Coral Reef Studies, The University of Queensland, St Lucia Campus, Brisbane, Qld 4072, Australia. ${ }^{19}$ UNEP- World Conservation Monitoring Centre, Cambridge, UK CB3 0DL. ${ }^{20}$ CBER - University College London, London, United Kingdom WC1E 6BT. ${ }^{21}$ WCPA-SSC Joint Task Force on Biodiversity and Protected Areas, International Union for the Conservation of Nature (IUCN), Quebec, Canada J9B 1T3. ${ }^{22}$ National Geographic Society, Washington, D.C. 20036, USA. *Current affiliations: Moore Center for Science, Conservation International. Arlington, Virginia 22202 USA; George Mason University, Fairfax, Virginia 22030 USA. Correspondence to: dgill@ conservation.org
} 

9 and ecological outcomes ${ }^{5-7}$.

Awareness of human impacts upon global marine biodiversity has spurred the largest expansion of marine protected areas (MPAs) in history ${ }^{1,2}$. As part of the 2011 Convention on Biological Diversity (CBD) Aichi Targets, 193 countries agreed to "effectively and equitably" manage 10\% of coastal and marine areas within marine protected areas and "other effective area-based conservation measures" by $2020^{3}$. A $10 \%$ conservation target for MPAs has also been included within Goal 14 of the United Nations Sustainable Development Goals (SDGs) ${ }^{4}$. Yet despite recent advances towards these coverage targets (currently $4.1 \%^{2}$ ), the efficacy and equity of many MPAs remain uncertain ${ }^{2}$; evidence suggests that MPAs often fail to deliver positive social

It is assumed that MPAs that are effectively regulated and actively managed through equitable and inclusive decision-making approaches are more likely to meet ecological and social goals than those that are merely legislated on paper ('paper parks') and those with exclusionary decision-making ${ }^{8-10}$. However, research linking the efficacy and equity of MPA management processes to conservation outcomes lies mostly in theory and select local-scale case studies ${ }^{11}$. This is largely due to a lack of a globally representative dataset on MPA management ${ }^{12}$ and lack of counterfactuals to infer ecological outcomes in the absence of MPAs ${ }^{13,14}$.

We constructed a global database of management and ecological data from 433 and 218 MPAs (respectively) to document and examine linkages between MPA management processes and conservation outcomes. Our dataset included MPAs from every tropical and temperate ocean basin, ranging in size from 0.006 to $989,836 \mathrm{~km}^{2}$, and span diverse social, political and biophysical contexts. First, to assess the efficacy and equity of MPA management processes, we drew on empirically-supported governance and management theories ${ }^{10,15-17}$ (Supplementary Table 1 and Extended Data Fig. 1) to identify key management process indicators from 433 
MPAs. We extracted data on these indicators from three widely applied survey instruments (Supplementary Table 2) that provided qualitative, Likert-scaled scores on questions posed to MPA stakeholders concerning MPA management activities and capacities ${ }^{18}$. From these, we defined binary thresholds for effective management based on the scoring criteria and alignment with social theory (Supplementary Tables 1 and 3). Second, to measure ecological impacts ( $n=218$ MPAs), we compiled MPA outcome data extracted from published studies ${ }^{5}(n=40$ MPAs) and transect or site level observations from unpublished regional and global datasets (Supplementary Table 2 and Extended Data Fig. 2; n=178 MPAs). For the unpublished ecological data, we calculated logged response ratios (lnRR) of mean fish biomass per unit area inside MPA sites relative to statistically matched control sites (i.e., pre-establishment and/or outside MPA; Methods). Finally, we investigated the relationship between management processes and ecological impacts in 62 MPAs where both management and ecological data were available. We used random forest and linear mixed effects models to identify important management predictors of ecological outcomes, while accounting for other factors known to impact fish responses to protection (e.g., MPA age and size ${ }^{7,19,20}$; Methods and Supplementary material).

\section{MPA management processes}

MPA management processes varied widely, with many of the 433 MPAs failing to meet thresholds for effective management (Fig. 1a). While the majority of MPAs were legally gazetted (79\%) and had appropriate regulations regarding resource use (69\%), most MPAs also reportedly made little to no use of scientific monitoring (biological, social or management) to inform management (13\%). Many also reported limited capacity, with $65 \%$ of MPAs reporting 
that their budget was inadequate for basic management needs and $91 \%$ stating that staff capacity (sufficient (on-site) staff capacity/numbers) was inadequate or below optimum.

Most MPAs were state managed (80\%), with the remaining either co-managed or managed by non-state actors (e.g., NGOs, local communities; Fig. 1a). Inclusive decision-making arrangements were reported in $51 \%$ of MPAs and were more common in shared/non-state managed MPAs than those managed solely by state agencies $(\mathrm{p}<0.001$; Extended Data Fig. 3).

Management processes were largely consistent across geographic contexts (Fig. 1b). In Oceania, however, devolved and inclusive management was more common and relatively few MPAs were legally gazetted. Where data were available for all indicators (excluding non-state management; n=277 MPAs), only $21 \%$ of MPAs met more than half of the nine thresholds, and only five MPAs (2\%) met all nine thresholds (Supplementary Table 7). Twenty-two MPAs (8\%) failed to meet any of the threshold levels for effective and equitable management.

\section{MPA ecological outcomes}

MPAs on average had positive, but variable, impacts on fish populations. We observed positive responses to protection in $71 \%$ of the 218 MPAs with fish biomass data. On average, fish biomass was 1.6 times higher in MPAs than in matched non-MPA areas (average logged response ratios $(\mathrm{LnRR})=0.47+0.96 \mathrm{SD})$. Positive responses were observed across almost all geographies and habitats (Fig. 2), consistent with other analyses ${ }^{5,20}$. Response ratios varied marginally by latitudinal zone $(F=2.963, p=0.087$; Fig. $2 b)$ and significantly among habitats $(F=$ 6.403, p<0.001; Fig. 2c) and continental regions $(F=5.284, p<0.001$; Fig. 2d). MPAs or MPA zones where all fishing was prohibited ("no-take") had higher response ratios than MPAs/zones where fishing was permitted (“multi-use") by almost two-fold ( $\mathrm{t}=2.24, \mathrm{p}=0.026$; Extended Data 
Fig. 4). Nonetheless, on average, we observed positive response ratios in both multi-use MPAs and MPA zones that prohibited fishing. Responses in prohibited fishing areas were lower than in some previous studies (for example, $82 \%$ increase in fish biomass in our study vs. $387 \%$ reported elsewhere ${ }^{5}$ ), likely due in part to the statistical matching approach, which reduced the observable biases arising from non-random MPA placement.

\section{Linking MPA management and outcomes}

We then explored the relationships between management processes and ecological impacts in MPAs for which we had both management and ecological data (62 MPAs in 24 countries), while accounting for other significant MPA and contextual attributes (e.g., MPA age, size, ocean conditions; Supplementary Table 4). In these MPAs, adequate staff capacity was the most important factor in explaining fish responses to MPA protection (Fig. 3a). Budget capacity was the second most important management variable and had similar performance in other analyses (Supplementary Table 9); however, budget data were only available in 43 MPAs. Clearly defined boundaries, MPA age and size, location (ecoregion, country), mean chlorophyll concentration, and mean shore distance were also identified as important by the conditional inference forest models (Fig 3a).

Our results demonstrate that effective biodiversity conservation is not simply a function of environmental (e.g., ocean conditions) or MPA features (e.g., MPA size, age, fishing regulations), but is also heavily dependent on available capacity (Fig. 3). Staff capacity was by far the most important explanatory variable in our study, accounting for approximately $19 \%$ of the variation in ecological outcomes $(\mathrm{p}<0.001)$. Qualitative examination of the MPA management data indicated that additional staff resources were needed to support monitoring, enforcement, administration, community engagement and sustainable tourism activities (inter 
alia). Though specific capacity needs varied among MPAs, biomass response ratios were on average 2.9 times greater in MPAs reporting adequate staff capacity than those MPAs reporting inadequate or no capacity (Fig. 3b). Where data were available (n=43 MPAs), we observed a significant relationship between budget capacity and ecological impacts (Supplementary Table 9), even after we removed potential outlying data (Extended Data Fig. 5a; $n=42$ MPAs; $t=2.55$; $\mathrm{p}=0.019$ ). Budget capacity was also significantly correlated with staff capacity (Spearman's rho $0.35, \mathrm{p}<0.001$ ), and both capacity variables were positively correlated with many of the other management variables (Extended Data Fig. 6). Thus, the effectiveness of many other key management processes may be limited by available human and financial capacity.

In addition to staff capacity, clearly defined boundaries and appropriate regulations were significantly correlated with ecological outcomes (Extended Data Fig. 7). However, the predictive strength of these two variables was sensitive to the modelling approach. Other management variables theorized to foster sustainable outcomes in common pool resources (e.g., inclusive decision making, monitoring of the resource and users ${ }^{15}$ ) were not significantly related to ecological performance (Fig. 3a and Supplementary Table 9), a finding consistent with some previous studies ${ }^{21,22}$. A possible explanation is that these described processes have stronger, more direct effects on resource users than on resource conditions ${ }^{22}$ or that the indicators used in management assessments may imperfectly measure the governance and management processes from common pool resource theory ${ }^{23}$ (e.g., Ostrom's design principles ${ }^{15}$ ).

Like others, we found that non-management factors such as MPA age and size also shape MPA ecological impacts (Fig 3a) ${ }^{7,19,20}$. Although we observed a significant difference in ecological impacts between prohibited fishing and multi-use zones (Extended Data Fig. 4), fishing regulations were not significant in our sample of 62 MPAs while controlling for (or interacting 
114 with) other factors (Fig 3a. and Supplementary Table 9). Other variables, such as proximity to

115 shore and chlorophyll concentration (a potential proxy for ocean productivity ${ }^{24}$ but also for

116 reduced coastal water quality at extremely high levels ${ }^{25}$ ), were negatively correlated with fish

117 biomass. This suggests that land-based stressors may be shaping impacts inside nearshore MPAs,

118 as noted in other work ${ }^{25,26}$. Differences in variable constructs among studies may partially

119 explain observed differences in our results from previous work. For example, a recent study that

120 found "enforcement" to be a significant factor" measured the enforcement construct as a

121 combination of compliance, community support, and enforcement activities, whereas our study

122 focused on management inputs into enforcement activities.

123

124

125

126

127

\section{Assessing MPA efficacy and equity}

We drew on social theory (Supplementary Table 1) to identify aspects of MPA management hypothesized to be important for ecological outcomes, independent of many of the MPA and site features also known to affect MPA performance (e.g. MPA age, size ${ }^{7,19}$ ). Our theory-based analytic framework (Extended Data Fig. 1 and Supplementary Table 1) provides a robust, replicable approach to measuring the procedural and substantive efficacy and equity of protected areas. In particular, integrated use of impact evaluation methodologies and indicators derived from widely used MPA monitoring tools permits us to make novel, evidence-based inferences of conservation impacts at a global scale ${ }^{27}$. Despite uneven geographic distribution and limited data on some indicators, this study represents one of the most comprehensive assessments of MPA management and ecological outcomes to date. While the ecological data center heavily on areas in the Northern Atlantic, U.S. Pacific, and Australia, the available management data are more dominant in other geographies (e.g., Africa, Europe, Southeast Asia), particularly in the developing world. These spatial incongruities limit the overlap between our ecological and 
137 management datasets ( $\mathrm{n}=62$ MPAs), but collectively provide a broad view on global MPA

138 performance.

139 Given data availability, our research focused on the efficacy and equity of MPA management

140 processes and, as an indicator of substantive efficacy, the ecological impacts of MPAs on fish

141 populations. We lacked sufficient data on other taxa to assess other ecological indicators of

142 substantive efficacy. We were also unable to measure the substantive social impacts of MPAs,

143 particularly substantive equity; the spatial and temporal resolutions of relevant data were too

144 coarse or geographically-limited to assess these impacts globally. Our research highlights a need

145 for contemporaneous social, ecological, and management data in order to fill these remaining

146 knowledge gaps and explore synergies and tradeoffs among the procedural and substantive

147 outcomes of conservation. To guide conservation policy, future research should examine

148 interactions between MPAs and other management measures (e.g., fisheries management), as

149 well as site-specific MPA capacity needs.

150 Achieving global conservation targets

151 As we approach the CBD and SDG milestone year of 2020, the global conservation community

152 and many governments will continue to invest heavily in MPA expansion ${ }^{1}$. Although many

153 MPAs with low management capacity in our sample had positive ecological impacts, in general

154 the magnitude of ecological impacts was strongly linked to the available human and financial

155 capacity for MPA management. Given the widespread shortfall in staff capacity that we

156 document worldwide (Fig. 4), inadequate capacity appears to compromise the ecological

157 performance of many MPAs. Adequate capacity is likely to be even more critical in the future, as

158 increasing anthropogenic pressures on marine resources necessitate more resilient marine

159 ecosystems and corresponding management regimes. For effective and equitable management to 

increased investment has the potential to dilute already scarce resources across a larger management area, weakening management and leaving many marine habitats and species at risk.

\section{3}

With such a high dependence on under-resourced MPAs to meet current and future conservation and sustainable development goals ${ }^{3,4}$, investment in MPA capacity development would potentially result in high returns on investment for both people and nature ${ }^{28}$.

\section{REFERENCES}

1. Lubchenco, J. \& Grorud-Colvert, K. Making waves: The science and politics of ocean protection. Science. 350, 382-383 (2015).

2. UNEP-WCMC \& IUCN. Protected Planet Report 2016. (United Nations Environment Programme (UNEP) World Conservation Monitoring Centre (UNEP-WCMC) and International Union for Conservation of Nature (IUCN), 2016).

3. Secretariat of the CBD. Aichi Target 11. Decision X/2. Convention on Biological Diversity (2011).

4. UN. United Nations Sustainable Development Goal 14: Conserve and sustainably use the oceans, seas and marine resources. (2016). Available at: http://www.un.org/sustainabledevelopment/oceans/.

5. Lester, S. E. et al. Biological effects within no-take marine reserves: a global synthesis. Mar. Ecol. Prog. Ser. 384, 33-46 (2009).

6. Mascia, M. B., Claus, C. A. \& Naidoo, R. Impacts of marine protected areas on fishing communities. Conserv. Biol. 24, 1424-1429 (2010).

7. Edgar, G. J. et al. Global conservation outcomes depend on marine protected areas with five key features. Nature 506, 216-220 (2014).

8. Pollnac, R. B., Crawford, B. R. \& Gorospe, M. L. G. Discovering factors that influence the success of community-based marine protected areas in the Visayas, Philippines. Ocean Coast. Manag. 44, 683-710 (2001).

9. Basurto, X., Blanco, E., Nenadovic, M. \& Vollan, B. Integrating simultaneous prosocial and antisocial behavior into theories of collective action. Sci. Adv. 2, e1501220-e1501220 (2016).

10. Mascia, M. B. in Marine Reserves: A Guide to Science, Design, and Use (eds. Sobel, J. \& Dahlgren, C.) 164-186 (Island Press, 2004).

11. Pollnac, R. et al. Marine reserves as linked social-ecological systems. Proc. Natl. Acad. Sci. U. S. A. 107, 18262-18265 (2010).

12. Fox, H. E. et al. How Are Our MPAs Doing? Challenges in assessing global patterns in marine protected area performance. Coast. Manag. 42, 207-226 (2014).

13. Ferraro, P. J. Counterfactual thinking and impact evaluation in environmental policy. New Dir. Eval. 2009, 75-84 (2009).

14. Ahmadia, G. N. et al. Integrating impact evaluation in the design and implementation of monitoring marine protected areas. Philos. Trans. R. Soc. B Biol. Sci. 370, 20140275 (2015).

15. Ostrom, E. Governing the commons: the evolution of institutions for collective action. (Cambridge University Press, 1990). 
16. Scianna, C., Niccolini, F., Gaines, S. D. \& Guidetti, P. 'Organization Science': A new prospective to assess marine protected areas effectiveness. Ocean Coast. Manag. 116, 443-448 (2015).

17. Hockings, M. et al. Evaluating Effectiveness: A framework for assessing management effectiveness of protected areas. 2nd edition. (International Union for Conservation of Nature (IUCN), 2006).

18. Coad, L. et al. Measuring impact of protected area management interventions: current and future use of the Global Database of Protected Area Management Effectiveness. Philos. Trans. R. Soc. B Biol. Sci. 370, 20140281 (2015).

19. Claudet, J. et al. Marine reserves: size and age do matter. Ecol. Lett. 11, 481-489 (2008).

20. Lester, S. \& Halpern, B. Biological responses in marine no-take reserves versus partially protected areas. Mar. Ecol. Prog. Ser. 367, 49-56 (2008).

21. McClanahan, T. R., Marnane, M. J., Cinner, J. E. \& Kiene, W. E. A comparison of marine protected areas and alternative approaches to coral-reef management. Curr. Biol. 16, 1408-1413 (2006).

22. Cinner, J. E. et al. Comanagement of coral reef social-ecological systems. Proc. Natl. Acad. Sci. 109, 5219$5222(2012)$.

23. Nolte, C. \& Agrawal, A. Linking management effectiveness indicators to observed effects of protected areas on fire occurrence in the Amazon rainforest. Conserv. Biol. 27, 155-165 (2013).

24. Williams, I. D. et al. Human, Oceanographic and Habitat Drivers of Central and Western Pacific Coral Reef Fish Assemblages. PLoS One 10, e0120516 (2015).

25. Wenger, A. S. et al. Effects of reduced water quality on coral reefs in and out of no-take marine reserves. Conserv. Biol. 30, 142-153 (2016).

26. Advani, S., Rix, L. N., Aherne, D. M., Alwany, M. A. \& Bailey, D. M. Distance from a Fishing Community Explains Fish Abundance in a No-Take Zone with Weak Compliance. PLoS One 10, e0126098 (2015).

27. Ferraro, P. J. \& Pressey, R. L. Measuring the difference made by conservation initiatives: protected areas and their environmental and social impacts. Philos. Trans. R. Soc. B Biol. Sci. 370, 20140270 (2015).

28. Watson, J. E. M., Dudley, N., Segan, D. B. \& Hockings, M. The performance and potential of protected areas. Nature 515, 67-73 (2014). 


\section{Figure legends}

\section{Figure 1 | Percent of MPAs exceeding or falling below threshold values for indicators of effective and equitable} management processes. Values shown for $\mathbf{a}$, all MPAs ( $\mathrm{n}=433$ MPAs) and $\mathbf{b}$, by continent. Dark blue bars (right) indicate the proportion of MPAs with scores at or above the threshold value, light blue bars (left) indicate the proportion below the threshold. Details on indicators, scores and threshold values in Supplementary Tables 1 and 3.

Figure 2 | Fish biomass response ratios (natural log scale) for 218 MPAs. a, Global variation in ln response ratio (lnRR) values. Positive response ratios (blue) indicate MPAs with greater biomass inside MPA relative to matched non-MPA areas. Negative values are in red. Base map sourced from ${ }^{50}$. b-d, Mean response ratios (dot) and $95 \%$ confidence interval (error bars) for multi-use areas (light blue) and areas where fishing is prohibited (dark blue) in 260 zones in 218 MPAs shown by $\mathbf{b}$, latitudinal zone, $\mathbf{c}$, habitat, and $\mathbf{d}$, continental regions. Y-axis parentheses indicate the number of MPAs/zones (multi-use, fishing prohibited respectively).

Figure 3 | Relationship between MPA management processes and ecological impacts. a, Random forest variable importance measures for management (dark blue bars) and other (non-management; light grey bars) variables as they relate to ecological impacts in $62 \mathrm{MPAs}$. Importance measures exceeding the red dashed line are considered non-random. b, Average fish biomass response ratios (dot) and 95\% confidence interval (error bars) for multi-use areas (light blue) and areas where fishing is prohibited (dark blue) by reported staff capacity (excluding MPAs with intermediate scores $(n=4)$ ). Y-axis parentheses indicate the number of MPAs/zones (multi-use, fishing prohibited respectively). Additional bivariate plots in Extended Data Fig. 5.

Figure 4 | Reported level of MPA staff capacity. MPAs reporting adequate (dark blue), inadequate or below optimum (blue) and no (light blue) staff capacity in their most recent management assessments where spatial data were available ( $\mathrm{n}=243$ MPAs; excludes MPAs with intermediate scores $(\mathrm{n}=5)$ ). Base map sourced from ${ }^{50}$. 


\section{METHODS}

MPA attribute and zone information. MPA geospatial and attribute data (i.e., location, shape/boundaries, age, area, fishing regulations) were sourced from the October 2015 version of the World Database on Protected Areas (WDPA) ${ }^{29}$ as well as other regional and international MPA datasets (see Supplement Information). Where possible, these data were supplemented and/or validated using scientific publications, reports, other official government and nongovernment sources, the ecological data providers, and local expert knowledge (Supplementary Table 4). For the purpose of this study, "fishing prohibited" refers to an MPA or zone within an MPA that prohibits any type of fishing activity, including subsistence and recreational fishing.

MPA management data. Data on MPA management processes were sourced from three management assessment tools: Management Effectiveness Tracking Tool (METT) ${ }^{30}$, the World Bank MPA Score Card ${ }^{31}$, and the NOAA Coral Reef Conservation Program's (CRCP) MPA Management Assessment Checklist ${ }^{32}$ (Supplementary Table 2).

Management indicator scores were rescaled to ensure construct validity between the assessments (Supplementary Table 3). To assist with the interpretation of the different scoring levels and criteria, we defined binary thresholds for each indicator based on the description of the scoring levels and social theory (Supplementary Tables 1 and 3). These thresholds were for descriptive purposes only; we used the rescaled indicator scores (as described in Supplementary Table 3) in the statistical models. For MPAs that had multiple management assessments, we used the most recent assessments available for describing the status of management processes in MPAs worldwide (e.g., for results in Fig. 1). For the models testing relationships with ecological outcomes, we used the assessment that was closest in time to when the ecological surveys were done, preferably before the ecological data were collected. If no assessment was available before 
271 the ecological surveys, we chose the one closest in time after the survey. When there was more

272 than one assessment in the same year we used the median score. There were a few cases of

273 survey respondents reporting non-integer scores (e.g. 2.5) or cases when such scores arose from

274 calculating the median value for a specific year (see Extended Data Fig. 8). No rounding was

275 carried out on non-integer scores, however; MPAs with these non-integer values were excluded

276 from maps and graphics (Fig. 3b and Fig. 4) to simplify interpretation.

277 Ecological impact data. We derived ecological data on marine fish populations from seven

278 independent global and regional datasets, with the majority comprising species-level data from

279 underwater visual census (UVC) surveys on coral or rocky nearshore reefs (Supplementary Table

280 2), and the remainder coming from meta-analyses (Lester et al. 2009; Lester \& Halpern 2008).

281 For the UVC data (15,978 survey sites), biomass represents the total biomass of all recorded fish

282 species, averaged across all transects at each site (grams per $100 \mathrm{~m}^{2}$ ). Variations in sample

283 methods meant that the choice of recorded species varied between datasets; therefore response

284 ratios were never calculated among surveys from different datasets. Biomass values were

285 calculated by the data providers or the authors using the individual body lengths and allometric

286 length-weight data obtained either from the data provider or from FishBase (www.fishbase.org).

287 Isolating MPA impacts. We identify MPA impacts by comparing MPA survey sites to

288 comparable non-MPA sites (outside MPA boundaries and/or before establishment) and

289 calculating logged response ratios (LnRR). Here we use statistical matching and other

290 procedures (described below) to account for: i) selection biases in MPA placement; ii) spatial-

291 temporal dynamics of fish response to protection (e.g. spill-over, recovery time) and; iii) other

292 biological, social and physical factors that can affect fish populations ${ }^{14}$. 
312 differences.

Effective assessment of MPA impacts necessitates the isolation of response to protection (MPA treatment) from other confounding factors ${ }^{33}$. Statistical matching allows us to develop a functional counterfactual by using the same factors that determine where MPAs are placed (e.g. opportunity costs for fishing) to select control sites ${ }^{13,14}$. Other factors that explain variation in fish populations (e.g., habitat, depth, wave energy) can also be used as covariates in the matching process. This assumes that, conditional on confounding covariates (both observed and unobserved), the control and treatment sites are inter-exchangeable, that is, from the same population ${ }^{34}$. Thus, with appropriate metrics or proxies of potentially confounding variables, control (non-MPA) and treatment (MPA) survey sites can be appropriately matched, with the majority of the remaining variation in the differences between the two groups attributable to the treatment (MPA protection) effect ${ }^{35}$.

Controlling for spill-over and response time-lags. Before matching, we removed survey sites that might confound the measurement of impacts. To account for (spatial) spillover effects, only control survey sites greater than one kilometer away from an established MPA boundary were used in the analysis (1,116 control sites removed). Despite many individual species having larger home ranges ${ }^{36,37}$, a review of studies examining spillover effects of marine reserves by Halpern et $\mathrm{al}^{38}$ indicates that one kilometer is a sufficient distance beyond which most population-level MPA effects can no longer be detected. Any spillover effects present in sites beyond this range will result in a more conservative estimate of MPA effects as it will reduce the inside-outside To account for time lags in fish response to protection, we assigned a survey site to an MPA only

314 if the MPA was established for at least three years. Initial detectable responses to protection can 315 be quite rapid (e.g. $1.5-2$ years ${ }^{39}, 1-3$ years ${ }^{40}, 2-5$ years ${ }^{41}$ ) and three years appeared to be 
sufficient time for MPA effects to become detectable. All sites within an MPA less than three years old were not used as MPA (treatment) sites ( $\mathrm{n}=579$ sites). All survey sites located within the boundaries of an MPA before the first (complete) year of MPA establishment were treated as "before" (control) sites given that a protection response is unlikely to occur within so short a period of time ( $\mathrm{n}=123$ sites or $3.0 \%$ of 4,125 control sites).

After removing the above mentioned sites and sites with ambiguous locations $(\mathrm{n}=1,882$ sites total), we proceeded with matching on 14,096 survey sites, comprising 9,971 treatment (MPA) and 4,125 (non-MPA) control sites.

Matching to control for observable bias. Based on existing literature on MPA site-selection biases and factors affecting variation in fish populations, Supplementary Table 5 describes the variables compiled for each survey site and used in the matching process. We performed multivariate matching using the Matching package $4.9-0{ }^{35}$ in the statistical software $\mathrm{R} v$ 3.2.3 ${ }^{42}$. We assessed the performance of various matching iterations using the post-matching covariate match balance outputs (Supplementary Table 6) and quantile-quantile plots. Here we attempted to reduce the standardized mean differences between covariates for control (non-MPA) and treatment (MPA) to below 5\%, which is considered appropriate for studies assessing casual inference ${ }^{43}$. We chose nearest neighbor multivariate matching algorithms (based on Mahalanobis distances), as they performed better than propensity score algorithms for our data. As there were fewer control than treatment sites, we matched with replacement, and allowed multiple control sites to be matched to each treatment site. Matching with replacement prevents ordering effects and allows the algorithm to choose the best available match from the entire population of control sites. Allowing multiple treatment-control matches reduces the influence of outliers by increasing the number of matched pairs. For our data, matching two controls to each 
treatment site $(2: 1$ ratio) resulted in lower standardized mean differences in treatment-control covariates than 1:1 matching, or using higher ratios (e.g. 3:1,4:1). All covariates carried equal weight, however covariate 'calipers' were used to ensure lower differences between the treatment and control sites for select covariates ${ }^{14}$ (see Supplementary Table 5). To help determine appropriate calipers, we used random forest models and partial dependency plots to explore the relationship between each covariate and fish biomass (using no-take sites to control for fishing effects). These were useful in determining both the strength of the relationship between the covariate and fish biomass, and to identify asymptotic peaks beyond which the covariate has no effect (e.g. shore distance appeared to have little effect on fish biomass beyond $20 \mathrm{~km})$. Calipers improved the quality of the matching, but reduced the overall number of possible matches; 2,335 (23\%) treatment (MPA) sites were dropped due to failure to find appropriate controls to match the treatment sites. Some of these drops were due to failure to find an appropriate control site within the same country or close in time to match with the treatment site. This resulted in 15,821 matched pairs for 7,636 treatment sites in 178 MPAs. These matched pairs were used to derive (natural log) response ratios for total fish biomass, which were averaged to the MPA level (Extended Data Fig. 8k).

We used Rosenbaum's bounds sensitivity analysis to assess the vulnerability of our MPA treatment effects to unobserved biases (i.e., factors not included in our list of matching covariates that could confound our estimates of MPA impact ${ }^{34,44}$ ). Rosenbaum's sensitivity bounds do not indicate whether or not such biases exist, but merely the potential for such a bias to influence our findings. When assessing the sensitivity of our estimates of MPA impacts on fish biomass to an unobserved variable, we find that if such a variable was able to change the odds of a site being protected by a factor $(\Gamma)$ of 1.35 , it would confound our estimate of impact. While $\Gamma=1.35$ 
suggests some sensitivity in our findings to potential unobserved bias, there is no evidence to suggest such a bias exists. Our extensive list of observed covariates (Supplementary Table 5) were identified through expert knowledge, the scientific literature, and available primary and secondary data as key factors that affect both MPA participation and outcomes. Further, covariates that remained significant after matching (e.g. shore distance, chlorophyll) were controlled for in subsequent models (Supplementary Table 9).

We supplemented the matched UVC data ( $\mathrm{n}=178$ MPAs) with MPA-level fish biomass ratios from the Lester et al. datasets ${ }^{5,20}$ ( $n=40$ MPAs), which comprise response ratios derived from 149 peer-reviewed publications that examine the ecological effects of areas where fishing is prohibited (marine reserves or no-take areas) and areas where fishing is allowed but restricted (multi-use). Where data were available for an MPA in both the Lester et al. and matched datasets ( $\mathrm{n}=11$ MPAs), we chose the latter. No matching was required for the Lester et al. data as response ratios were already formulated by the authors in their meta-analysis. The final ecological dataset totaled 218 MPAs (see Extended Data Fig. 2 for data compilation steps).

Management and ecological data analysis. We used random forests with conditional inference trees $^{45}$ to identify the management processes (Supplementary Table 4) that best explained the variation in ecological impacts (n=62 MPAs). Random forests account for higher-order interactions and nonlinear relationships between predictors, and do not require many of the strict assumptions of linear parametric models that are difficult to meet ${ }^{46}$. These qualities make random forests an ideal approach for our analysis, where many interacting and non-linear relationships among management processes, MPA attributes, and ecological outcomes are expected ${ }^{11}$. Random forests are also able to effectively estimate variable importance in "small $\mathrm{n}$, large p" models and models with missing data ${ }^{46,47}$. 
385 In this study, we used the $R$ "party" package v1.0-25 ${ }^{48}$ to estimate the relative variable 386 importance of the ten management indicators using the log fish biomass response ratios as the 387 response variable and the metric for ecological impacts. In addition to the management 388 indicators, we also included other non-management variables as predictors in the model. Many 389 of these were identified in the literature as being important in explaining variability in fish 390 populations and MPA ecological outcomes (MPA age, MPA size, fishing regulations) ${ }^{7,19,20}$, and 391 include many of the variables used in the matching process (mean MPA depth, shore distance, 392 market distance, human population density, chlorophyll, wave exposure, sea surface temperature, 393 ecoregion, country; Supplementary Table 5). This allowed us to assess the relative importance of 394 the management indicators as predictors, while accounting for (and allowing interactions with) 395 these potentially important non-management factors.

396 Given that we were investigating the MPA level effects of management, the MPA was 397 considered as the unit of analysis. Therefore all variables, including response ratios, were 398 averaged to the MPA level. All non-management predictors represent the MPA-level average of 399 the conditions at each fish survey site (e.g. mean depth represents the mean depth of the fish 400 survey sites in that MPA). All continuous predictors were transformed to the natural log scale to 401 reduce the effect of extreme outliers with the exception of depth which did not need to be 402 transformed. Proportion no-fishing represents the proportion of survey sites for an MPA sampled 403 from within a prohibited-fishing (no-take) zone (0: all multi-use, 1: all prohibited fishing). See 404 the Supplemental Information for more details on the procedures and variables used in the 405 random forest modelling.

406 We also ran a series of general linear mixed-effects models (Supplementary Table 9) to examine 407 the direction and strength of the relationships between each of the management indicators and 
408 ecological impacts. The linear mixed effects models allowed us to examine the predictor-

409 response relationships in a hierarchical model structure, while controlling for other important

410 non-management factors. These non-management variables were those identified as important in

411 the random forest models (mean chlorophyll, mean shore distance, mean MPA age, MPA size)

412 and those found to be important in the literature (i.e., fishing regulations: "proportion no

413 fishing"). For the hierarchical structure, we included a random intercept for country to account

414 for potential non-independence in the fish response to protection between MPAs in the same

415 country (e.g. MPAs managed by the same national agency). Including country as a random

416 intercept performed similarly to other random effect structures that account for spatial hierarchy

417 (see Supplementary Table 8). We used the $R$ "nlme" package v3.1-128 49 to implement the linear

418 mixed models and only included one management predictor in each model due to strong

419 correlation (Extended Data Fig. 6) and missing data amongst some of the predictor variables.

420 The results are shown in Supplementary Table 9. 
29. IUCN and UNEP-WCMC. The World Database on Protected Areas (WDPA). Accessed October 2015. (2015). Available at: www.protectedplanet.net. (Accessed: 1st October 2015)

30. Stolton, S. et al. Management Effectiveness Tracking Tool: Reporting progress in Protected areas sites; second edition. (World Bank/WWF Forest Alliance and WWF, 2007).

31. Staub, F. \& Hatziolos, M. E. Score Card to Assess Progress in Achieving Management effectiveness goals for Marine Protected Areas. (Prepared for the World Bank, 2004).

32. NOAA. NOAA Coral Reef Conservation Program MPA Management Assessment Checklist. (National Oceanic and Atmospheric Administration (NOAA), 2010).

33. Mora, C. \& Sale, P. Ongoing global biodiversity loss and the need to move beyond protected areas: a review of the technical and practical shortcomings of protected areas on land and sea. Mar. Ecol. Prog. Ser. 434, 251-266 (2011).

34. Rosenbaum, P. R. Design Sensitivity and Efficiency in Observational Studies. J. Am. Stat. Assoc. 105, 692 702 (2010).

35. Sekhon, J. S. Multivariate and Propensity Score Matching. J. Stat. Softw. 42, 1-52 (2011).

36. Alerstam, T., Hedenstrom, A. \& Akesson, S. Long-distance migration: evolution and determinants. Oikos 103, 247-260 (2003).

37. Green, A. L. et al. Larval dispersal and movement patterns of coral reef fishes, and implications for marine reserve network design. Biol. Rev. 90, 1215-1247 (2015).

38. Halpern, B. S., Lester, S. E. \& Kellner, J. B. Spillover from marine reserves and the replenishment of fished stocks. Environ. Conserv. 36, 268-276 (2010).

39. Russ, G. R. et al. Rapid increase in fish numbers follows creation of world's largest marine reserve network. 18, 514-515 (2006).

40. Halpern, B. S. \& Warner, R. R. Marine reserves have rapid and lasting effects. Ecol. Lett. 5, 361-366 (2002).

41. Gell, F. R. \& Roberts, C. M. Benefits beyond boundaries: the fishery effects of marine reserves. Trends Ecol. Evol. 18, 448-455 (2003).

42. R Development Core Team. R: A language and environment for statistical computing, version 3.2.3. (2015).

43. Caliendo, M. \& Kopeinig, S. Some practical guidance for the implementation of propensity score matching. J. Econ. Surv. 22, 31-72 (2008).

44. Keele, L. An overview of rbounds: An R package for Rosenbaum bounds sensitivity analysis with matched data. Citeseer 1-15 (2010).

45. Hothorn, T., Hornik, K. \& Zeileis, A. Unbiased recursive partitioning: A conditional inference framework. J. Comput. Graph. Stat. 15, 651-674 (2006).

46. Strobl, C., Malley, J. \& Tutz, G. An introduction to recursive partitioning: rationale, application, and characteristics of classification and regression trees, bagging, and random forests. Psychol. Methods 14, 323-348 (2009).

47. Hapfelmeier, A., Hothorn, T., Ulm, K. \& Strobl, C. A new variable importance measure for random forests with missing data. Stat. Comput. 24, 21-34 (2012).

48. Hothorn, T., Hornik, K., Strobl, C. \& Zeileis, A. Package 'Party': A Laboratory for Recursive Partytioning. $\mathrm{R}$ package version 3.1-128. (2015).

49. Pinheiro, J. C. \& Bates, D. M. Linear and nonlinear mixed-effects models, R package version 3.1-128. (2016). Available at: http://cran.r-project.org/web/packages/nlme/index.html. 
465

466

50. Sandvik, B. World Borders Dataset. (2016). Available at: http://thematicmapping.org/downloads/world_borders.php. 
Supplementary Information accompanies this paper. The majority of the MPA ecological and management data reported in this paper are tabulated in the online supplementary materials.

Acknowledgments: This research was supported by the National Socio-Environmental Synthesis Center (SESYNC) under funding received from the National Science Foundation DBI1052875, as part of the working group: Solving the Mystery of Marine Protected Area (MPA) Performance: Linking Governance, Conservation, Ecosystem Services and Human Well Being. David Gill was jointly supported by postdoctoral fellowships from the Luc Hoffmann Institute and SESYNC. We thank the following data providers: Atlantic Gulf Rapid Reef Assessment (AGRRA), Conservation International, Healthy Reefs Initiative, I. Williams (NOAA Coral Reef Ecosystem Program), NOAA Coral Reef Conservation Program, K. Knights (Global Database for Protected Area Management Effectiveness), G. Edgar and R. Stuart-Smith (Reef Life Surveys), The Nature Conservancy, Wildlife Conservation Society and the World Conservation Monitoring Centre. We also thank other members of the SESYNC MPA Pursuit team: A. Agrawal, G. Cid, A. Henshaw, I. Nur Hidayat, W. Liang, P. McConney, M. Nenadovic, J.E. Parks, B. Pomeroy, C. Strasser, M. Webster; and P. Marchand of SESYNC for scientific support.

Author contributions: H.E.F. and M.B.M. conceived the study. D.A.G. led the analysis and data compilation with the assistance of H.E.F., M.B.M., G.N.A., L.G., S.E.L., M.B., I.C., E.D., C.M.F., J.G., S.H., O.P.J., G.G., H.T., S.W. and S.W. C.M.F. prepared the maps. D.A.G., H.E.F., M.B.M., G.N.A., L.G., and S.E.L. wrote the manuscript with the input of all the other authors.

Competing financial interests: The authors declare no competing financial interests. 
489 Reprints and permission information is available online at

490 http://npg.nature.com/reprintsandpermissions/

491 Materials \& Correspondence: Correspondence and requests for materials should be addressed

492 to D.A.G. (dgill@ conservation.org). 


\section{Extended data legends}

Extended Data Figure 1 |Key domains and illustrative indicators for assessing management efficacy and equity. Indicators with asterisks are those that were used in this study. Details on indicator descriptions, sources and citations are located in Supplementary Table 1.

Extended Data Figure 2 | Sources and major steps in the data compilation and analysis. See Supplementary Table 2 for more details on data sources. *CRCP: Coral Reef Conservation Program

Extended Data Figure 3 | Percent of MPAs by managing authority exceeding or falling below threshold values for indicators of effective and equitable management processes. Details on indicators, scores and threshold values in Supporting Tables 1 and 3. Dark blue bars (right) indicate the proportion of MPAs with scores at or above the threshold value, light blue bars (left) indicate the proportion below the threshold. Scores are from the latest assessment year where data were available from 433 MPAs.

Extended Data Figure 4 | Mean response ratios (natural log scale) of fish biomass. Mean (dot) and 95\% confidence intervals (error bars) for areas where fishing is prohibited (dark blue) and multi-use MPA areas (light blue) in 254 zones in 218 MPAs.

Extended Data Figure 5 | Relationship between MPA-averaged fish biomass response ratios and key predictor variables used in the analysis of the relationship between MPA management processes and ecological impacts ( $\mathbf{n}<62$ MPAs). a-j, mean (black point) and 95\% confidence intervals (error bars) of the response ratios for each management score and indicator. Details on threshold levels and score descriptions in Supplementary Table 3. k-t, Smoothed LOESS lines (blue line) along with the standard error region (shaded area) for relationships with continuous variables. Number of MPAs in parentheses.

Extended Data Figure 6 | Spearman rank correlations amongst management indicators, national variables and other key variables (n=433 MPAs). Variables ordered using hierarchical clustering, displaying values for significant correlations only $(\mathrm{p}<0.05)$. Circle size and color indicate the correlative strength and direction (blue positive and red negative) respectively. Most of the management indicators for procedural efficacy were significantly correlated with each other (e.g. correlation coefficient for monitoring and management plan $=0.49$ ) 
National level variables (GDP, HDI) were poorly correlated with management indicators and were not included in this study. ENF: Adequate enforcement; BGT: Acceptable budget capacity; REG: Appropriate MPA regulations; MON: Monitoring informing management activities; MPL: Implementing existing management plan; BND: Clearly defined boundaries; LEG: Legally gazetted; STF: Adequate staff capacity/presence; IDM: Inclusive decisionmaking; DEV: Non-state/shared management; SIZ: MPA size $\left(\ln \left(\mathrm{km}^{2}\right)\right)$; AGE: MPA age (ln(years)); HDI: Human Development Index 2010; GDP: Gross Domestic Product per capita (ln(US\$ PPP)) 2013.

\section{Extended Data Figure 7 | Spearman rank correlations amongst fish metrics, management indicators, and} other key variables for the 62 MPAs used in the management and ecological data analysis. Circle size and color indicate the correlative strength and direction (blue positive and red negative) respectively. Variables ordered by type (i.e. ecological, management, etc.) and not hierarchical clusters, displaying values for significant correlations only (p<0.05). BIO: (ln) fish biomass response ratio; DEN: (ln) fish density response ratio; FSZ: (ln) fish mean size response ratio; RCH: (ln) fish species richness response ratio; DEV: Non-state/shared management; IDM: Inclusive decision-making; LEG: Legally gazetted; BND: Clearly defined boundaries; REG: Appropriate MPA regulations; ENF: Adequate enforcement; MON: Monitoring informing management activities; MPL: Implementing existing management plan; STF: Adequate staff capacity/presence; BGT: Acceptable budget capacity; NTZ: Proportion of survey sites for an MPA sampled from within a prohibited-fishing (no-take) zone; SIZ: MPA size $\left(\ln \left(\mathrm{km}^{2}\right)\right)$; AGE: MPA age (ln(years)); CHO: chlorophyll-a concentration $\left(\ln \left(\mathrm{mg} / \mathrm{m}^{3}\right)\right)$; SHR: Distance from shore $(\ln (\mathrm{km}))$.

\section{Extended Data Figure 8 | Frequency distribution of MPA-management, ecological and other key variables.}

White bars indicate the distribution of: $\mathbf{a - j}$, scores from the latest available management assessments ( $\leq \leq 433$ MPAs); k-n, MPAs where fish biomass data were available ( $n \leq 218$ MPAs). a-n, Grey bars indicate MPAs used in the analysis modeling the relationship between management processes and ecological impacts ( $\mathrm{n} \leq 62$ MPAs). Indicators for $\mathbf{b}$, inclusive decision-making and $\mathbf{g}$, enforcement have a maximum score of 2 . Non-integer values were reported scores by few managers, or represent the median value of multiple assessments in the latest year. $\mathbf{k}$, average MPA level response ratios (natural log scale) for fish biomass. l, proportion of survey sites for an MPA sampled from within a prohibited-fishing (no-take) zone (0: all multi-use area; 1 : all no-take/prohibited fishing area). m, MPA age 
(years between establishment and fish survey). $\mathbf{n}$, MPA size $\left(000 \mathrm{~km}^{2}\right)$. MPA age and size were transformed to the $\log$ scale for the analysis.

Extended Data Figure 9 | Random forest variable importance plots. Random forest variable importance measures for management (blue bars) and other (non-management; grey bars) variables as they relate to ecological impacts in 62 MPAs. Results from models with a, all management indicators (as shown in Fig. 3a in the main text) and $\mathbf{b}$, management indicators with few missing data and not highly correlated with other predictors (i.e. excluding legal status, acceptable budget, management plan, country and ecoregion). Only values greater than the red dashed line are considered to have non-random importance scores. Also shown in $\mathbf{c}$ and $\mathbf{d}$ are the predicted and observed response ratio values from the random forest models in $\mathbf{a}$ and $\mathbf{b}$ respectively, along with the linear fitted line (dashed blue line) and a smoothed LOESS line along with the standard error region (grey line and shaded area). $\mathrm{R}^{2}$ values for the linear fit are also shown. 